\title{
DISTRIBUIÇÃO TERRITORIAL DOS INVESTIMENTOS SOCIAIS PRIVADOS E SUA RELAÇÃO COM CARACTERİSTICAS LOCAIS
}

\section{Guilherme Hirata}

\section{// resumo}

O investimento social privado é um importante mecanismo para promoção, complementação e até substituição de políticas públicas que visam combater a pobreza, a desigualdade e a falta de oportunidades que atingem a população mais vulnerável. Este artigo realiza três análises quantitativas com dados brasileiros. A primeira verifica a distribuição territorial de projetos sociais privados nas áreas de educação e mercado de trabalho. A segunda compara características dos municípios que recebem projetos com os que não recebem. E a terceira analisa a relação entre características dos municípios e a quantidade de projetos para os municípios que implementam ao menos um projeto. As características locais consideradas refletem o nível de desenvolvimento econômico, a qualidade da educação, a desigualdade de renda e o nível de atividade econômica. A verificação da distribuição territorial ocorre por meio de contagem do número de projetos existentes, tanto em termos absolutos quanto em relação ao tamanho da população. A análise da associação entre existência e quantidade de projetos e as características locais é realizada por meio de regressão linear múltipla. Os resultados indicam que municípios mais desenvolvidos em estados mais desiguais têm mais chance de receber investimentos sociais privados. Por outro lado, a implementação de uma quantidade maior ou menor de projetos não está associada às características locais, como qualidade da educação e desigualdade.

palavras-chave: investimento social privado | educação | mercado de trabalho. 


\section{OS ARTIGOS GIFE}

A série Artigos GIFE publica reflexões e análises de pesquisadores brasileiros de diferentes áreas do saber com o objetivo de estimular, ampliar e disseminar a produção de conhecimento qualificado sobre o campo da filantropia, do investimento social privado e da sociedade civil no Brasil.

A partir da premissa de que a produção de conhecimento sobre esses temas tem um papel fundamental na expansão, diversificação e qualificação da ação pública pela sociedade, a iniciativa também pretende contribuir para ampliar as conexões entre o setor, as universidades e os centros de pesquisa, promovendo e apoiando o envolvimento de novos atores com a temática e a circulação ampliada de ideias em torno dela.

Este texto integra a quarta edição dos Artigos GIFE, que propõe a publicação mensal de artigos selecionados por meio de chamada pública e convite a autores especialistas em temáticas específicas e relevantes para o setor, buscando apoiar e fomentar a produção de conhecimento sobre o investimento social no Brasil.

O Grupo de Institutos Fundações e Empresas (GIFE) é uma organização sem fins lucrativos que reúne associados de origem empresarial, familiar ou independente que investem em projetos de interesse público. Criado em 1989 como grupo de troca e colaboração e institucionalizado em 1995 por 25 organizações, tornou-se referência no campo da filantropia e do investimento social privado no Brasil.

Nesses mais de 20 anos de atuação, tem contribuído para o fortalecimento de práticas e ações a serviço do bem comum no país, trabalhando para expandir, qualificar e fortalecer o investimento social privado, diversificar e ampliar atores e recursos, criando referências e estimulando boas práticas de gestão, bem como articulando o setor com a sociedade e a agenda pública. Atualmente, o GIFE reúne mais de 150 associados que, somados, aportam mais de R\$ 3 bilhões por ano em projetos próprios e no apoio a projetos de terceiros. 


\section{INTRODUÇÃO}

De acordo com o Grupo de Institutos Fundações e Empresas, considerando 133 associados que participaram do Censo 2018, houve um aporte anual de 3 bilhões de reais em uma diversa gama de projetos sociais, abrangendo diversas áreas (GIFE, 2019). Isso evidencia o volume de recursos disponíveis para investimentos sociais que complementa ou mesmo substitui a atuação governamental em determinados casos.

Este artigo analisa, de forma geral, a distribuição territorial dos projetos realizados pelos associados do GIFE e a relaciona com as características socioeconômicas e educacionais dos municípios em que são realizados. Os projetos analisados atuam nas áreas de educação e mercado de trabalho. As características locais referem-se ao nível de desenvolvimento econômico, à qualidade da educação, à desigualdade de renda e ao nível de atividade econômica. Especificamente, o objetivo do artigo é gerar uma reflexão sobre o destino dos investimentos e contribuir para melhorar a eficiência dos aportes a partir de uma perspectiva mais global. Lugares mais desiguais têm maior probabilidade de receber projetos na área de mercado de trabalho que qualifiquem a mão-de-obra? Quanto pior a qualidade do ensino, maior o número de projetos executados? Essas são algumas das perguntas que serão respondidas.

As perguntas formuladas refletem o contexto em que o artigo está inserido, a saber, o papel do investimento privado. A implantação de projetos sociais tem capacidade para impactar de fato a vida das pessoas. A questão é saber se as vidas impactadas são aquelas em locais onde o governo já atua, onde há necessidade de complementação ou onde o governo não alcança, seja por dificuldade operacional, seja simplesmente por abandono, involuntário ou não. Cada investidor tem estratégias de atuação e objetivos distintos. Mas até que ponto a atuação pulverizada de muitos investidores, cada qual com suas características, cada um buscando seu espaço, de fato ajuda a resolver o problema atacado? Considerando todos os projetos realizados, é eficiente investir sem uma análise cuidadosa de o quê e onde já foi feito, de o que funciona e como funciona?

O artigo divide-se em mais quatro seções além desta introdução. A primeira traz uma breve discussão sobre investimento privado. Em seguida, a seção 2 trata da fonte de dados, descreve os procedimentos para a seleção da amostra de projetos, a escolha das variáveis utilizadas e apresenta a metodologia implementada. A seção 3 reporta os resultados da análise, seguida das considerações finais. 


\section{INVESTIMENTO SOCIAL PRIVADO: OS DOIS LADOS DA MOEDA}

O investimento social privado tem dois atores principais, o investidor e o beneficiário. A literatura sobre o tema abrange aspectos associados a eles, seja separadamente, seja conjuntamente.

Do lado dos beneficiários de projetos sociais privados, Aoqui e Santos (2019) tratam da procura por investimentos para a periferia de São Paulo. Por meio de encontros com lideranças locais, os autores identificaram dois componentes-chave que dificultam e/ou limitam o acesso a investimentos sociais privados: fatores culturais e comportamentais - compreensão da realidade em que o projeto está inserido - e fatores técnicos - como o cuidado com a linguagem verbal e não-verbal.

Do lado do investidor, Nogueira, Abdal e Campos (2019) analisam a cultura de doação no país e argumentam que um grande obstáculo para realização de doações é a falta de informação a respeito de diversos aspectos, desde o desconhecimento do que seriam direitos humanos até estudos que mostrem evidências de impacto.

Tentando preencher a lacuna da falta de informações, Addy et al. (2019) propõem a criação de um indicador que os autores chamam de impact multiple of money (IMM), que nada mais é que uma medida do retorno social de cada unidade monetária a ser investida em determinado projeto. Esse tipo de informação é relevante tanto para investidores quanto para potenciais beneficiários de um dado projeto a ser realizado em outros locais. Os autores argumentam que a ausência de uma medida mais objetiva de impacto associada aos projetos em busca de financiamento faz com que investidores se baseiem somente em informações sobre objetivos e processos, sem ter referência a respeito dos reais benefícios que tais projetos podem trazer para a sociedade.

Vale ressaltar que há um relacionamento entre o setor público e o investimento social privado. Lopes e Bueno (2012) investigam a relação da União com o investimento social privado ao analisar as transferências federais para entidades privadas sem fins lucrativos. Os dados reportados mostram que o volume de recursos transferidos a cada ano quase dobrou de 1999 a 2010, alcançando mais de 4,1 bilhões de reais no último ano da série $\left(6,6\right.$ bilhões de reais, a preços de $\left.2020^{1}\right)$. Aproximadamente $15 \%$ desses recursos foram direcionados às áreas de educação e trabalho. De fato, a aproximação e a maior colaboração entre setores público e privado têm sido reportados na literatura, com ações baseadas na ideia de complementaridade na provisão dos serviços oferecidos à população (NOGUEIRA e SCHOMMER, 2009; SANTOS e MENDONÇA, 2019).

O presente artigo contribui tanto para a literatura quanto para os investidores, ao ampliar o conjunto de informações que podem ser úteis na tomada de decisão da realização de investimentos. Ainda que o volume de recursos não seja modesto, há sempre mais projetos em busca de recursos do que recursos disponíveis, tendo o investidor a missão de identificar onde investir.

\section{FONTE DE DADOS, DEFINIÇÕES E METODOLOGIA}

Este artigo conta com diversas fontes de dados. A principal base de dados foi disponibilizada pela equipe do GIFE a pedido do autor (GIFE, 2020). Trata-se de uma lista com iniciativas de associados respondentes do Censo GIFE 2018², em execução naquele ano, e suas respectivas informações, como descrição, local de atuação, instituição responsável e financiadores (GIFE, 2019). Tais iniciativas continuaram a ser atualizadas desde então na plataforma Base de Projetos do Mosaico, portal de dados do investimento social.

1 Valor deflacionado pelo Índice de Preços ao Consumidor Amplo (IPCA).

2 Trata-se de uma base de dados em formato de planilha Excel que apresenta a listagem dos projetos/ programas das organizações respondentes do Censo GIFE 2018 que autorizaram a divulgação de suas iniciativas na plataforma Base de Projetos do portal Mosaico. Os dados foram coletados entre junho e agosto de 2019, durante o Censo, e incluem apenas projetos/ programas que estavam em execução em 2018. 
O estudo foi feito com uma listagem de 597 projetos. Foram excluídos os projetos que: i) não tinham descrição, por não ser possível identificar a área de atuação; e ii) não indicavam o local de execução ou indicavam todos os municípios do país. No segundo caso, há projetos em plataformas digitais que potencialmente podem alcançar todo o território, mas não há como comprovar que todos os municípios realmente são atingidos. Após as exclusões, obteve-se 250 projetos.

Como a listagem não incluía a área de atuação dos projetos, foi feita uma classificação própria a partir de sua descrição, dividindo-os em nove áreas: educação, mercado de trabalho, cidadania, cultura e esporte, desenvolvimento local, meio-ambiente, saúde, social e outros. Os projetos de educação envolviam basicamente a qualificação de gestores e professores e programas voltados a melhorar o desempenho dos alunos ou direcionados a crianças em creches e pré-escolas. Os de mercado de trabalho, de modo geral, promoviam capacitação e treinamento de mão-de-obra, com ou sem suporte para encontrar emprego.

São duas as variáveis construídas a partir da base de dados de projetos. A primeira indica se existe ou não algum projeto em educação ou mercado de trabalho em cada município. Se um mesmo projeto é executado em dois municípios, o projeto foi contado duas vezes, uma para cada município. Esta variável permite analisar a possibilidade de superar a barreira de entrada e estabelecer o primeiro projeto. Por vezes, o primeiro contato com o local onde se pretende investir é a etapa mais delicada. Assim, comparar as características dos locais onde existem projetos em execução com as de municípios que não recebem projeto algum pode trazer insights que ajudem a identificar e ampliar o número de locais que recebem investimento.

A segunda variável traz o número de projetos de cada área em cada município, construída somente para municípios com pelo menos um projeto existente. Isso possibilita verificar se municípios que recebem muitos projetos são diferentes daqueles que recebem poucos projetos. Neste caso, não está em questão a superação da barreira de entrada mencionada anteriormente. Utilizou-se o número de projetos para cada 10 mil habitantes.

Para analisar a distribuição territorial de projetos relacionados à educação e ao mercado de trabalho e sua relação com as características socioeconômicas e educacionais dos locais onde são executados, o trabalho foi feito em três etapas. A primeira apresenta a distribuição geográfica dos projetos por região e unidade da federação (UF). A segunda compara as características locais de municípios com e sem projetos em execução. E a terceira avalia a relação entre quantidade de projetos e as características locais.

Para a segunda e terceira etapas, as variáveis que medem características locais são: qualidade da educação, desenvolvimento econômico, taxa de desemprego e desigualdade de renda. Cada variável busca medir quantitativamente uma característica local. A escolha das variáveis derivou da disponibilidade de informações e de sua relevância e clareza, todas de fácil interpretação.

A qualidade da educação é estimada por meio do desempenho escolar dos alunos da rede pública de cada município. Os dados provêm do Instituto Nacional de Estudos e Pesquisas Educacionais Anísio Teixeira (INEP, 2018), órgão vinculado ao Ministério da Educação. Especificamente, são utilizadas as notas da Prova Brasil, um exame nacional de matemática e língua portuguesa para estudantes de $5^{\circ}$ e $9^{\circ}$ anos do ensino fundamental. Todas as escolas públicas com ao menos 20 alunos matriculados nas séries-alvo são elegíveis a participar. Os dados utilizados foram de 2017, ano mais recente disponível. Utiliza-se a nota padronizada calculada pelo Inep, que é uma média das provas de matemática e língua portuguesa, e que varia de 0 a 10. Há uma nota padronizada para o $5^{\circ}$ e $9^{\circ}$ anos. A variável final é a média das duas notas.

O produto interno bruto (PIB) per capita, disponibilizado pelo Instituto Brasileiro de Geografia e Estatística (IBGE, 2019), é utilizado para medir o nível de desenvolvimento econômico. Essa variável está mensurada em milhares de reais.

A taxa de desemprego para indivíduos com 14 anos ou mais é utilizada como medida de atividade econômica. A taxa é calculada a partir dos dados da Pesquisa Nacional por Amostra de Domicílios (PNAD) Contínua, coletados pelo IBGE (IBGE, 2020). Trata-se de uma pesquisa trimestral contendo informações sociodemográficas e de mercado de trabalho, tais como idade, sexo, escolaridade, 
participação no mercado de trabalho e rendimentos. Foi utilizada a edição do $4^{\circ}$ trimestre de 2019. Devido ao desenho amostral da pesquisa, não é possível estimar a taxa de desemprego para municípios. Assim, utilizou-se a taxa de desemprego para os estados.

A PNAD Contínua também foi utilizada para estimar a desigualdade de renda. A partir da informação de rendimentos coletada pela pesquisa, que inclui rendimentos de trabalho e outras fontes (aluguel, pensão, benefícios assistenciais etc.), foi estimado o índice de Gini de desigualdade de renda. O índice mede o quão distante a distribuição de renda entre os brasileiros está de uma situação hipotética em que todos recebem a mesma renda. O índice varia de 0 a 1 , sendo que, quanto maior o valor, maior é a desigualdade de renda. Novamente, não é possível estimar a medida para municípios devido às limitações dos dados.

Para a segunda e terceira etapas, foram usados modelos de regressão linear múltipla que associam a presença e a quantidade de projetos às características locais. No caso da relação entre presença de projetos e características locais, os resultados das regressões indicam a chance de existir ao menos um projeto no município. Intuitivamente, tomando a variável de PIB per capita como exemplo, o modelo estatístico permite saber se municípios com menor PIB per capita apresentam maior probabilidade de ter projetos na área de educação. Para o caso de quantidade de projetos, é possível avaliar se quanto menor o PIB per capita de um município, maior é a quantidade de projetos implementados.

\section{QUE DIZEM OS DADOS A RESPEITO DA DISTRIBUIÇÃO DO INVESTIMENTO PRIVADO}

Esta seção apresenta os resultados do artigo. Primeiramente, apresenta-se a distribuição dos projetos executados entre estados e regiões. A seguir, discute-se a relação entre existência de projetos e características locais. Finalmente, para municípios onde há projetos em execução, analisa-se se há diferença entre municípios com menor e maior número de projetos.

\subsection{Distribuição geográfica dos projetos}

A primeira etapa da pesquisa verificou a distribuição dos projetos entre UF. Na Tabela 1, a primeira e a segunda colunas dizem respeito aos projetos de educação, ao passo que a terceira e a quarta tratam dos projetos de mercado de trabalho. Apresenta-se, para cada região, o subtotal de projetos executados e a média de projetos por 1 milhão de habitantes.

Como observado na coluna 1, a região Sudeste é a que apresenta o maior número de projetos de educação (386), com o estado de São Paulo recebendo o maior número de projetos do país, 269 (ou $33 \%$ do total de projetos do país, 698). Dois estados se destacam fora da região Sudeste: Pernambuco, com 104 projetos, e Pará, com 80.

A distribuição dos projetos de mercado de trabalho (coluna 3) segue o mesmo padrão. A maior parte se encontra nos estados do Sudeste, com um total de 120 projetos (ou 33\% do total). Amazonas e Maranhão são os que mais recebem projetos em suas respectivas regiões. O destaque negativo é a ausência de projetos no Acre e no Amapá.

As colunas 2 e 4 da Tabela 1 levam em consideração o tamanho da população para analisar a quantidade de projetos, indicando o número de projetos por milhão de habitantes. Isso ajuda a analisar o quadro a partir de outro ponto de vista. Ao mesmo tempo em que um estado pode ter mais projetos, pode ter também um público-alvo maior. Ou seja, 10 projetos pode ser pouco para um estado com muita população, mas pode muito para um estado com pouca população.

Desse ponto de vista, a distribuição de projetos fica mais equilibrada territorialmente. Para projetos de educação, Pernambuco e Pará passam a ser os estados com mais projetos relativamente às suas respectivas populações, enquanto estados das regiões Norte e Centro-Oeste se aproximam dos demais. Já para os projetos de mercado de trabalho, Amazonas mantém-se em destaque, apresentando 14,70 projetos para cada um milhão de habitantes, número 10 vezes maior que o observado para São Paulo. Com essa medida alternativa, quase todos os estados do Nordeste também contam com relativamente mais projetos do que São Paulo. 
Tabela 1 - Número de projetos de educação e de mercado de trabalho por estado

\begin{tabular}{|c|c|c|c|c|c|}
\hline \multirow[b]{3}{*}{ Região } & \multirow[b]{3}{*}{ UF } & (1) & (2) & (3) & (4) \\
\hline & & \multicolumn{2}{|c|}{ Projetos de educação } & \multicolumn{2}{|c|}{ Projetos de mercado de trabalho } \\
\hline & & n. absoluto & $\begin{array}{l}\text { n. por } 1 \text { milhão } \\
\text { de habitantes }\end{array}$ & n. absoluto & $\begin{array}{l}\text { n. por } 1 \text { milhão } \\
\text { de habitantes }\end{array}$ \\
\hline \multirow[t]{8}{*}{ Norte } & RO & 1 & 0,57 & 2 & 1,14 \\
\hline & $\mathrm{AC}$ & 1 & 1,15 & 0 & 0,00 \\
\hline & AM & 17 & 4,17 & 60 & 14,70 \\
\hline & RR & 1 & 1,73 & 1 & 1,73 \\
\hline & PA & 80 & 9,40 & 6 & 0,70 \\
\hline & AP & 2 & 2,41 & 0 & 0,00 \\
\hline & TO & 8 & 5,14 & 1 & 0,64 \\
\hline & SUBTOTAL & 109 & 3,51 & 70 & 2,70 \\
\hline \multirow[t]{10}{*}{ Nordeste } & $\mathrm{MA}$ & 6 & 0,85 & 24 & 3,41 \\
\hline & $\mathrm{Pl}$ & 4 & 1,23 & 11 & 3,37 \\
\hline & CE & 6 & 0,66 & 11 & 1,21 \\
\hline & RN & 5 & 1,44 & 10 & 2,87 \\
\hline & PB & 16 & 4,00 & 11 & 2,75 \\
\hline & $P E$ & 104 & 10,95 & 19 & 2,00 \\
\hline & $A L$ & 21 & 6,32 & 8 & 2,41 \\
\hline & SE & 15 & 6,58 & 11 & 4,83 \\
\hline & BA & 17 & 1,15 & 13 & 0,88 \\
\hline & SUBTOTAL & 194 & 3,69 & 118 & 2,64 \\
\hline \multirow[t]{5}{*}{ Sudeste } & MG & 50 & 2,38 & 35 & 1,66 \\
\hline & ES & 14 & 3,52 & 4 & 1,01 \\
\hline & RJ & 53 & 3,09 & 15 & 0,87 \\
\hline & SP & 269 & 5,91 & 66 & 1,45 \\
\hline & SUBTOTAL & 386 & 3,73 & 120 & 1,25 \\
\hline \multirow[t]{4}{*}{ Sul } & PR & 49 & 4,32 & 15 & 1,32 \\
\hline & SC & 34 & 4,81 & 9 & 1,27 \\
\hline & $\mathrm{RS}$ & 18 & 1,59 & 9 & 0,79 \\
\hline & SUBTOTAL & 101 & 3,57 & 33 & 1,13 \\
\hline \multirow[t]{5}{*}{ Centro-oeste } & MS & 7 & 2,55 & 1 & 0,36 \\
\hline & MT & 6 & 1,74 & 6 & 1,74 \\
\hline & GO & 10 & 1,44 & 7 & 1,01 \\
\hline & DF & 2 & 0,67 & 4 & 1,34 \\
\hline & SUBTOTAL & 25 & 1,60 & 18 & 1,11 \\
\hline TOTAL BRASIL & & 698 & 3,32 & 305 & 2,05 \\
\hline
\end{tabular}


Essa primeira análise indica para onde estão indo os projetos de educação e mercado de trabalho. Quando se leva em consideração o tamanho da população, observa-se que todos os estados do Centro-Oeste estão abaixo da média do país em projetos de educação; nas demais regiões, há estados acima e abaixo da média. Em mercado de trabalho, a maior parte dos estados do Nordeste está acima da média, juntamente com o Amazonas da região Norte; todos os estados do Sul, Sudeste e Centro-Oeste estão abaixo da média. A resposta que se busca nas próximas etapas é se há alguma relação entre a alocação de projetos e as características dos locais onde estão sendo implantados. A próxima seção verifica se os locais onde existe ao menos um projeto são diferentes dos locais em que nenhum projeto foi implantado, desta vez observando os municípios.

\subsection{Presença de projetos}

Esta seção discute a relação entre características socioeconômicas e educacionais locais e presença de projetos de educação e mercado de trabalho. A Tabela 2 mostra os coeficientes para cada variável, bem como o intervalo de confiança. As três primeiras linhas referem-se a projetos de educação, enquanto as demais tratam de projetos de mercado de trabalho.

\section{Tabela 2 - Relação entre existência de projetos de educação e mercado de trabalho e características socioeconômicas e educacionais locais}

\begin{tabular}{|c|c|c|c|}
\hline Projeto & Característica & Coeficiente & Intervalo de confiança \\
\hline \multirow[t]{3}{*}{ Educação } & Prova Brasil & 0,003 & $(-0,011 ; 0,016)$ \\
\hline & PIB per capita & 0,004 & $(0,004 ; 0,005)$ \\
\hline & Desigualdade & 1,228 & $(0,983 ; 1,472)$ \\
\hline \multirow[t]{4}{*}{ Mercado } & Prova Brasil & $-0,001$ & $(-0,010 ; 0,009)$ \\
\hline & PIB per capita & 0,002 & $(0,002 ; 0,003)$ \\
\hline & Desigualdade & 0,830 & $(0,572 ; 1,088)$ \\
\hline & Desemprego & $-0,076$ & $(-0,414 ; 0,261)$ \\
\hline
\end{tabular}

Fonte: Prova Brasil, INEP (2018); PIB, IBGE, (2019); desigualdade - PNAD, IBGE (2020); desemprego - PNAD, IBGE (2020). Elaboração do autor. Notas: Intervalo de confiança de 95\% baseado na distribuição t de Student. R-quadrado da regressão para projetos de educação: 0,0369. R-quadrado da regressão para projetos de mercado de trabalho: 0,0279. Número de observações em cada regressão: 5.546 . Não foram detectados problemas de multicolinearidade via correlação de Pearson. Não foi testada a autocorrelação por se tratar de dados cross-section.

Inicialmente, são discutidos os resultados para projetos de educação. Em primeiro lugar, observa-se que não há relação entre qualidade da educação (Prova Brasil) e existência de projetos. O intervalo de confiança para o coeficiente, cujo valor é 0,003 , inclui o valor zero. Ou seja, em média, a chance de um município receber ao menos um projeto de educação não está relacionada à qualidade da educação existente no município. Em outras palavras, municípios nos quais se observa que a educação não vai bem não têm maior chance de receber um projeto que municípios onde há educação de melhor qualidade.

Em segundo lugar, municípios menos desenvolvidos, isto é, com PIB per capita mais baixo, têm menor chance de receber projetos. No entanto, a diferença não é tão grande assim. A cada 5.000 reais acrescidos no PIB per capita de um município, há uma probabilidade de receber projetos de educação 2 
pontos percentuais maior $(0,004 \times 5)^{3}$. Isso é equivalente a dizer que um município mais desenvolvido que metade dos municípios brasileiros tem apenas 2 pontos percentuais de maior probabilidade de receber o primeiro projeto de educação - financiado pelo investimento social privado da base do GIFE (2020) - que outro que está aproximadamente entre os 15\% menos desenvolvidos do país.

Os resultados também dizem que municípios localizados em estados mais desiguais têm maior probabilidade de receber algum projeto. Nesse caso, não são necessariamente os municípios mais desiguais de cada estado. Ao juntar essa evidência com a do parágrafo anterior, os dados sugerem que os municípios mais desenvolvidos de estados mais desiguais têm maior chance de receber projetos de educação.

Passando agora para os projetos de mercado de trabalho, o cenário geral é parecido com o observado para projetos de educação. Lugares com educação de pior qualidade não têm mais chance de receber projetos. Vale lembrar que, entre os projetos de mercado de trabalho, há aqueles que qualificam mão de obra.

Por outro lado, municípios mais desenvolvidos e aqueles localizados em estados mais desiguais têm maior chance de receber projetos. Porém, novamente, essa relação com o nível de desenvolvimento econômico é fraca: um maior PIB per capita de 5.000 reais aumenta a probabilidade de receber um ou mais projetos de mercado de trabalho em apenas 1 ponto percentual $(0,002 \times 5$, coluna 4$)$. Ou seja, não há grande diferença. A existência de projetos parece não depender do desenvolvimento econômico do município.

Finalmente, observa-se também que não há relação entre existência de projetos em mercado de trabalho e a atividade econômica medida pela taxa de desemprego. O intervalo de confiança inclui o valor zero, fazendo com que o valor estimado não seja significante estatisticamente. Em todo caso, como o desempenho econômico do estado pode não representar a realidade para alguns municípios, para alguns em particular a relação pode ser diferente, mas não é possível captá-la com os dados disponíveis.

Portanto, em média, ainda que a relação seja fraca, os projetos de mercado de trabalho tendem a ser realizados em lugares mais desenvolvidos e mais desiguais, mas não nos estados em que o desemprego é maior. Com isso, encerra-se a análise a respeito da presença de projetos. Os fatores que influenciam a quantidade de projetos nos municípios em que há ao menos um projeto é o tema da próxima seção.

\subsection{Quantidade de projetos}

Esta seção compara as características dos municípios por quantidade de projetos existentes. Os resultados referem-se à quantidade relativa de projetos, ou seja, ao número de projetos por 10.000 habitantes ${ }^{4}$. Vale lembrar que são 250 projetos ao todo, sendo que um mesmo projeto pode estar presente em mais de um município, podendo, portanto, ser contabilizado mais de uma vez. Os municípios que não têm projetos não foram incluídos nesta análise, que compreende 625 municípios com projetos de educação e $261 \mathrm{com}$ projetos de mercado de trabalho. A Tabela 3 apresenta os resultados no mesmo formato da Tabela 2, sendo a interpretação dos coeficientes estimados distinta.

\footnotetext{
3 Uma vez que o PIB per capita é medido em milhares de reais.

4. Na Tabela 1, como a análise foi feita para os estados, a relação era para cada 1 milhão de habitantes.
} 


\section{Tabela 3 - Relação entre quantidade relativa de projetos em educação e mercado de trabalho e características locais iniciais e contemporâneas}

\begin{tabular}{|c|c|c|c|}
\hline Projeto & Característica & Coeficiente & Intervalo de confiança \\
\hline \multirow[t]{3}{*}{ Educação } & Prova Brasil & 0,091 & $(-0,029 ; 0,211)$ \\
\hline & PIB per capita & $-0,002$ & $(-0,006 ; 0,002)$ \\
\hline & Desigualdade & $-1,012$ & $(-3,023 ; 0,999)$ \\
\hline \multirow[t]{4}{*}{ Mercado } & Prova Brasil & $-0,118$ & $(-0,293 ; 0,058)$ \\
\hline & PIB per capita & $-0,007$ & $(-0,012 ;-0,002)$ \\
\hline & Desigualdade & 2,220 & $(-1,134 ; 5,575)$ \\
\hline & Desemprego & $-3,619$ & $(-9,105 ; 1,868)$ \\
\hline
\end{tabular}

Fonte: Prova Brasil, INEP (2018); PIB, IBGE, (2019); desigualdade - PNAD, IBGE (2020); desemprego - PNAD, IBGE (2020). Elaboração do autor. Notas: Intervalo de Confiança de 95\% baseado na distribuicão t de Student. R-quadrado da regressão para projetos de educacão: 0,0072. R-quadrado da regressão para projetos de mercado de trabalho: 0,0727. Número de observações: educação: 625; mercado de trabalho: 261 . Não foram detectados problemas de multicolinearidade via correlação de Pearson. Não foi testada a autocorrelação por se tratar de dados cross-section.

Considere-se primeiramente os projetos de educação. Observando os intervalos de confiança para as três variáveis, todos incluem o valor zero. Isso significa que nenhum coeficiente é estatisticamente significante. Ou seja, a evidência diz que não há qualquer relação entre as características socioeconômicas e educacionais locais e a quantidade de projetos realizados. Um município sem educação de qualidade não recebe mais projetos que um município com educação de boa qualidade. Do mesmo modo, um município menos desenvolvido recebe tantos projetos quanto um município mais desenvolvido.

Para o número de projetos em mercado de trabalho, o cenário é semelhante. Qualidade da educação no município e desigualdade no estado não parecem influenciar o direcionamento de esforços para esse tipo de projeto naquele território. Tampouco há relação com o nível de desemprego no estado. Apesar de o coeficiente estimado ser negativo no último caso, o intervalo de confiança inclui o zero. Novamente, a realidade do município pode ser diferente da observada no estado como um todo, mas a evidência traz indícios de que a implantação de projetos não acompanha o cenário econômico.

A única característica que tem alguma relação com a quantidade de projetos é o nível de desenvolvimento econômico: quanto menor o PIB per capita, maior é a quantidade de projetos de mercado de trabalho. Entretanto, observa-se que a relação é fraca. Um município com PIB per capita 10 mil reais inferior apresenta somente 0,07 projetos a mais para cada 10 mil habitantes.

As evidências desta seção sugerem não haver relação entre as características dos municípios e a quantidade de projetos em andamento, sejam eles de educação ou mercado de trabalho. Isso equivale a dizer que municípios menos desenvolvidos recebem uma quantidade de projetos semelhante ao que se observa em municípios mais desenvolvidos. 


\section{CONSIDERAÇÕES FINAIS}

A análise realizada a partir das características locais sugere que o aporte de recursos privados em investimento social são iniciativas que carecem de uma perspectiva mais global. O estudo indicou que os municípios menos desenvolvidos têm menores chances de obter projetos em educação e mercado de trabalho. Indicou também que a quantidade de projetos não está relacionada a características locais que levam em conta desenvolvimento econômico, qualidade da educação e desigualdade de renda. É até natural que isso aconteça em um cenário em que cada investidor tenha seus ideais, buscando promover projetos de seu interesse ao seu alcance. Contudo, há investidores que atuam nacionalmente, ao invés de regionalmente ou localmente. Estariam eles investindo de forma eficiente?

O setor privado, que clama por um setor público mais eficiente, precisaria pensar em soluções que revertam os resultados encontrados neste artigo. Haveria um ganho para a sociedade, por exemplo, se houvesse ao menos um banco de dados público que registrasse de forma uniforme e padronizada os projetos desenvolvidos, bem como seus resultados e avaliações de impacto externas, se for o caso. Ainda que cada investidor tenha seus métodos e objetivos particulares, um dos principais meios de se combater a pobreza e a desigualdade é via promoção de acúmulo de capital humano, e isso não ocorre se o investimento, público ou privado, não chega aos lugares onde o capital humano é mais escasso. Todos terem acesso às informações básicas dos projetos em andamento do país é uma condição necessária para uma alocação de recursos mais eficiente.

O artigo tem diversas limitações evidenciadas ao longo do texto e, certamente, análises mais rigorosas são necessárias para corroborar ou não os resultados apresentados. Somente com mais dados e um maior volume de estudos científicos é que se terá um cenário mais claro a respeito do investimento social privado no Brasil e de sua contribuição para a sociedade.Finalmente, observa-se também que não há relação entre existência de projetos em mercado de trabalho e a atividade econômica medida pela taxa de desemprego. O intervalo de confiança inclui o valor zero, fazendo com que o valor estimado não seja significante estatisticamente. Em todo caso, como o desempenho econômico do estado pode não representar a realidade para alguns municípios, para alguns em particular a relação pode ser diferente, mas não é possível captá-la com os dados disponíveis. 


\section{REFERÊNCIAS}

ADDY, Chris; CHORENGEL, Maya; COLLINS, Mariah; ETZEL, Michael. Calculating the value of impact investing. Harvard Business Review, January-February, p. 102-109, 2019.

AOQUI, Cássio; SANTOS, Diana Mendes dos. Novas narrativas para o investimento social e acesso a recursos nas periferias. Artigos GIFE, v. 1, n. 2, 2019. Disponível em: https://sinapse.gife.org.br/ download/novas-narrativas-para-o-investimento-social-e-acesso-a-recursos-nas-periferias. Acesso em: 1 de julho de 2020.

GIFE. Censo GIFE 2018. 2019. Disponível em: https://sinapse.gife.org.br/download/censo-gife-2018. Acesso em: 13 de julho de 2020.

GIFE. [e-mail]. Destinatário: Guilherme Hirata. 9 jan. 2020. 1 e-mail com arquivo xls que detalha projetos em andamento de associados GIFE no ano de 2018, a partir do Censo GIFE 2018.

IBGE - INSTITUTO BRASILEIRO DE GEOGRAFIA E ESTATÍSTICA. Produto interno bruto per capita. Rio de Janeiro: IBGE, 2019. Disponível em: https://www.ibge.gov.br/estatisticas/downloadsestatisticas.html. Acesso em: 13 jul. 2020.

IBGE - INSTITUTO BRASILEIRO DE GEOGRAFIA E ESTATÍSTICA. Pesquisa Nacional por Amostra de Domicílios Contínua. Rio de Janeiro: IBGE, 2020. Disponível em: https://www.ibge.gov. br/estatisticas/sociais/trabalho/9173-pesquisa-nacional-por-amostra-de-domicilios-continua-trimestral. html? edicao=26895\&t=microdados. Acesso em: 13 jul. 2020.

INEP - INSTITUTO NACIONAL DE ESTUDOS E PESQUISAS EDUCACIONAIS ANÍSIO TEIXEIRA. Microdados da Aneb e da Anresc 2017. Brasília: Inep, 2018. Disponível em:<http://portal.inep.gov.br/ basica-levantamentos-acessar>. Acesso em: 31 out. 2018.

LOPEZ, Felix G.; BUENO, Natália S. Transferências federais a entidades privadas sem fins lucrativos (1999-2010). IPEA, Texto para Discussão, n. 1.778, 2012.

NOGUEIRA, Fernando do Amaral; ABDAL, Alexandre; CAMPOS, Pedro Henrique. Práticas e percepções da doação entre a população de alta renda no Brasil. Artigos GIFE, v. 1, n. 2 2019. Disponível em: https://sinapse.gife.org.br/download/praticas-e-percepcoes-da-doacao-entre-a-populacao-de-altarenda-no-brasil. Acesso em: 1 de julho de 2020.

NOGUEIRA, Fernando do Amaral; SCHOMMER, Paula Chies. Quinze anos de investimento social privado no Brasil: Conceito e práticas em construção. In: XXXIII ENANPAD - Encontro Científico de Administração da ANPAD. Anais... São Paulo, 2019.

SANTOS, Beatriz Caroline Silvestre dos; MENDONÇA, Patrícia Maria E. de. Percepções e experiências dos gestores públicos sobre o alinhamento do investimento social privado às políticas públicas. Artigos GIFE, v.1, n.1 2019. Disponível em: https://sinapse.gife.org.br/download/percepcoes-e-experienciasdos-gestores-publicos-sobre-o-alinhamento-do-investimento-social-privado-as-politicas-publicas. Acesso em: 1 de julho de 2020. 


\section{Guilherme Hirata guilherme@idados.id}

Bacharel em Economia pela Universidade de São Paulo (USP), mestre em Economia pela Universidade Federal de Minas Gerais (UFMG) e doutor em Economia pela Pontifícia Universidade Católica do Rio de Janeiro (PUC-Rio). É coordenador da área de pesquisa em Educação da Consultoria IDados. Tem experiência em pesquisa em educação, mercado de trabalho e avaliação de impacto de políticas educacionais e sociais. Conta com publicações em revistas científicas nacionais e internacionais. Participou da elaboração do portal Data Zoom, tendo sido seu gerente operacional. Trabalhou como pesquisador associado do Centro Internacional de Políticas para o Crescimento Inclusivo (IPC-IG) e foi professor do Ibmec/RJ.

\section{AGRADECIMENTOS}

O autor agradece a Carolina Magosso, Gleice Guerra, João Batista Araujo e Oliveira e demais revisores desta edição dos Artigos GIFE, por sugestões e comentários, e a Isabella Helter, pela assistência prestada nesta pesquisa. Agradece ainda à Equipe GIFE por disponibilizar os dados para a realização deste artigo. 


\title{
artigos GIFE
}

Supervisão: José Marcelo Zacchi e Gustavo Bernardino

Coordenação: Carolina Magosso

Produção editorial: Gleice Regina Guerra

Apoio: Graziela Santiago

Comitê de seleção: Ana Lúcia D'Império Lima, Fábio Silva

Tsunoda, Laura Leal e Lívia Menezes Pagotto

Diagramação: Alastra, Comunica.

Impressão: Forma Certa

ISSN: 2674-8061

DOI: doi.org/10.33816/gife.20200202a4

(c) 2020 GIFE - Grupo de Institutos Fundações e Empresas

\section{(C) (i) ()}

Este material é disponibilizado sob a licenca Creative Commons Atribuição Não Comercial 4.0 Internacional. http://creativecommons.org/licenses/by-nc/4.0

As opiniões e análises expressas nesta publicação não necessariamente refletem as do GIFE.

\author{
Apoio institucional \\ Alana \\ Ford Foundation \\ Fundação Bradesco \\ Fundação Lemann \\ Fundação Tide Setubal \\ Instituto Unibanco \\ Laudes Foundation
}

